\title{
Ausrichtung der Forschungen der akademischen Mitarbeiter an der Theologischen Fakultät, Sektion Tarnów
}

\author{
Directions of the Scientific Research \\ Undertaken by Academic Staff of the Theology Faculty, \\ Section in Tarnów
}

\begin{abstract}
The $21^{\text {st }}$ century brings new challenges for the society and every human being. Although today's Europe is in the process of strengthening and enlarging its economic and political union, it seems to suffer from a profound crisis of values. Therefore, it is very important to have a proper perception and understanding of contemporary challenges for education and pedagogy. This might results in going through the effective learning processes, which enable a human being to function properly in his/her private and professional life. For all these reasons, the author of this article shows various aspects of scientific and didactic activities undertaken by the academic staff of the Faculty of Theology, Section in Tarnów, which is an integral part of the Pontifical University of John Paul II in Cracow. A short historical sketch of establishment of the Faculty of Theology, Section in Tarnów enable to embed the academic work of this Faculty in specific realities and conditions.
\end{abstract}

\section{Keywords}

Faculty of Theology, Section in Tarnów, academic staff, scientific research.

Das einundzwanzigste Jahrhundert bringt neuartige Herausforderungen für das Leben der gesamten Gesellschaft und jedes einzelnen Menschen mit sich. Darum ist es von außerordentlicher Bedeutung, die gegenwärtigen Herausforderungen im Bereich von Erziehung, Bildung und Pädagogik wahrzunehmen und zu verstehen 
sowie in diesem Kontext eine effektive Bildung und eine entsprechende Erziehung aufzunehmen, die es dem Menschen erlaubt, in seinem privaten und sozialen Leben richtiggehend zu bestehen und zu arbeiten. Das Statut der Päpstlichen Universität Johannes Paul II. in Krakau unterstreicht deshalb, dass ,die wissenschaftlichen Forschungen und die Lehre darauf ausgerichtet sein sollen, die Kenntnis der auf der christlichen Offenbarung basierenden Wahrheit zu vertiefen und in ihrem Licht die Probleme zu lösen, vor denen die Kirche steht"'. Unter anderem aus diesem Grund scheint es daher nur zu berechtigt, in dem vorliegenden Artikel Aspekte der Forschungen aufzuzeigen, welche die akademischen Mitarbeiter an der Theologischen Fakultät, Sektion Tarnów, durchführen. Nach einem Überblick über Geschichte und Grundsätzliches der Fakultät werden Forschungen vorgestellt und charakterisiert, an denen die Wissenschaftler an der Theologischen Fakultät, Sektion Tarnów, arbeiten.

\section{1. Überblick über die Geschichte der Theologischen Fakultät, Sektion Tarnów}

Die Theologische Fakultät, Sektion Tarnów, die aus dem Theologischen Institut hervorgegangen ist, kann bereits auf eine Tradition von nahezu zweihundert Jahren zurückblicken. Sie wurde jedoch erst im Jahr 1993 der Theologischen Fakultät der Päpstlichen Theologischen Akademie in Krakau (heute Päpstliche Universität Johannes Paul II. in Krakau) angegliedert und arbeitet in deren Strukturen. Da die entsprechende Anzahl an Studenten, eine ausreichende Zahl wissenschaftlicher Lehrstühle, die spezifische Materialbasis, die wissenschaftliche Grundlage sowie eine günstige Situierung vorhanden waren, wurde beschlossen, das Institut als selbstständige Fakultät zu formieren. Man nahm damals entsprechende Schritte auf, um das Prozedere anzupassen. In Folge dessen fasste der Senat der Päpstlichen Theologischen Akademie in Krakau den Beschluss, das Theologische Institut in Tarnów umzugestalten in die Theologische Fakultät, Sektion Tarnów (WTST), der Päpstlichen Theologischen Akademie in Krakau - heute Päpstliche Universität Johannes Paul II. in Krakau. Am 14. Mai 2004 genehmigte die Kongregation für das Katholische Bildungswesen die vorliegende Entscheidung und bestätigte das Statut sowie die Leitung der neu berufenen Fakultät. Folglich ist die Theologische Fakultät, Sektion Tarnów, organisatorisch gesehen eine Fakultät der Päpstlichen Universität Johannes Paul II. in Krakau. In ihrer wissenschaftlichen Arbeit folgt die Theologische Fakultät, Sektion Tarnów, den für eine Hochschule verpflichtenden

\footnotetext{
${ }^{1}$ Statut der Päpstlichen Universität Johannes Paul II. in Krakau, Nr. 107.
} 
Vorschriften, dem Statut der Päpstlichen Universität Johannes Paul II. in Krakau und den kirchlichen Anweisungen in den Dokumenten der Kirche, die hauptsächlich in der Apostolischen Konstitution Johannes Pauls II. „Sapientia Christiana” vom 15. April 1979, in dem Dokument „Ordinationes” und im Kodex des Kanonischen Rechts vom 25. Januar 1983 niedergelegt sind. Die Theologische Fakultät, Sektion Tarnów, bietet den Abschluss als Magister der Theologie und erhielt im Mai 2007 die Berechtigung, den akademischen Titel eines Doktors der Theologie zu verleihen. Es ist auch zu betonen, dass die Theologische Fakultät, Sektion Tarnów, in der Diözese Tarnów entstanden ist, die über eine reiche Tradition an wissenschaftlichen Forschungen ihrer Priester verfügt, was sich unter anderem darin ausdrückt, wie viele Titel und akademische Grade diese erworben haben. Denn aktuell haben von den lebenden Priestern der Diözese Tarnów etwa 140 einen Doktortitel, das sind ca. 10\% aller Priester. Darunter sind weitere 26 Priester mit Habilitation und zehn tragen den Titel eines Professors in nachfolgenden Disziplinen: Theologie, humanistische Wissenschaften, Rechtswissenschaften und Musik. An der Sektion der Theologischen Fakultät in Tarnów arbeitet jedoch nur ein Teil von ihnen, die übrigen sind an anderen Hochschulen und Universitäten in Polen tätig oder bereits emeritiert.

Die Theologische Fakultät, Sektion Tarnów, insbesondere das vorherige Theologische Institut in Tarnów, bildete seit seinem Bestehen Kleriker aus, die sich auf ihre Arbeit als Katecheten und Seelsorger in der Diözese Tarnów und der gesamten Kirche vorbereiten. Anfang der neunziger Jahre des 20. Jahrhunderts wurde auch für Laien die Möglichkeit geschaffen, dort das Studium in den Bereichen Katechese und Pastoral aufzunehmen. Im System der kostenlosen Studiengänge ermöglicht die WTST den Abschluss als Magister sowie als einzige Hochschule in Tarnów den Erwerb des Doktortitels, was derzeit für eine bedeutende Gruppe von Jugendlichen eine attraktive und die einzige zugängliche Chance darstellt. An der Theologischen Fakultät, Sektion Tarnów, der Päpstlichen Universität Johannes Paul II. in Krakau erhalten jedes Jahr zwischen neunzig und hundert Personen das Diplom als Magister der Theologie, auch konnten bis zum heutigen Tag bereits zwei Doktortitel verliehen werden. Der wissenschaftliche Betrieb wird in Tarnów in seiner Gesamtheit abgedeckt, da sich hier ebenfalls der Sitz der Fakultätsleitung und der Verwaltung befindet. Gegenwärtig besuchen die Theologische Fakultät, Sektion Tarnów, etwa 300 Studenten (100 Laien und 200 Kleriker).

An der Theologischen Fakultät, Sektion Tarnów, kann man Theologie mit dem Ziel des Magisterabschlusses studieren, dabei ist das System so angelegt, dass die Studiendauer für die Laien fünf und für die Kleriker sechs Jahre beträgt. Es werden drei Möglichkeiten der Spezialisierung angeboten: 
- im katechetisch-pastoralen Bereich (für Laienstudenten), ein fünfjähriges Magisterstudium;

- im sozial-karitativen Bereich (für Laienstudenten), ein fünfjähriges Magisterstudium;

- die Ausbildung zum Priester (für Kleriker, die sich auf die Arbeit als Seelsorger vorbereiten), ein sechsjähriges Magisterstudium.

\section{Forschungen der akademischen Mitarbeiter an der Theologischen Fakultät, Sektion Tarnów}

Die Theologische Fakultät, Sektion Tarnów, umfasst zehn Lehrstühle: Lehrstuhl für Philosophie, Lehrstuhl für Alttestamentliche Exegese, Lehrstuhl für Neutestamentliche Exegese, Lehrstuhl für Patrologie und Kirchengeschichte, Lehrstuhl für Fundamentaltheologie und vergleichende Religionswissenschaft (Ökumenismus), Lehrstuhl für Dogmatik, Lehrstuhl für Moraltheologie und Geistlichkeit, Lehrstuhl für Religionspädagogik und Katechese, Lehrstuhl für Kanonisches Recht und Lehrstuhl für Sozialwissenschaften, Pastoraltheologie, Liturgie und Homiletik. Jeder Lehrstuhl führt seine eigenen Forschungsarbeiten durch, die sich mit dem breiten Problemkreis vielfältiger Untersuchungen im Bereich von Philosophie und Theologie befassen. Darüber hinaus haben die Mitarbeiter der einzelnen Lehrstühle seit dem Bestehen als Sektion der Theologischen Fakultät in Tarnów ungefähr 50 Wissenschaftskonferenzen organisiert oder mitorganisiert.

Die Mitarbeiter des Lehrstuhls für Philosophie forschen auf den Gebieten der antiken, mittelalterlichen und neuzeitlichen Geschichte, der modernen Philosophie sowie auf dem breit gefassten philosophischen Fragenkreis im Rahmen von Psychologie, Philosophie, Lerntheorie, Logik und Naturphilosophie. Die von dem Lehrstuhl angebotenen Vorlesungen und didaktischen Seminare (Übungen zur Geschichte der Philosophie und der Logik) haben die Aufgabe, den historischen Hintergrund der Evolutionskonzeptionen und der philosophischen Strömungen allgemein nachzuzeichnen - insbesondere diejenigen, welche später in den theologischen Vorlesungen Verwendung finden. Die Analyse philosophischer Probleme unter vielfältigen Aspekten soll den Studenten auch dabei helfen, kritische und selbstständige Denkstrukturen zu entwickeln, die ihnen in späteren Jahren selbstständiges und erfolgreiches intellektuelles Arbeiten in jedem anderen Bereich der Wissenschaft und des Gesellschaftslebens ermöglichen werden. 
Der Lehrstuhl für Alttestamentliche Exegese befasst sich mit Arbeiten aus der Didaktik und mit wissenschaftlichen Forschungen. In Verbindung mit dem „Dzieło Biblijne im. Jana Pawła II” (Bibelwerk im Namen Johannes Pauls II.) werden Themen aus dem Alten Testament durch offene Bibelstudien für Laien und Geistliche gemeinverständlich behandelt. Im Rahmen der didaktischen Arbeiten führen die Mitarbeiter des Lehrstuhls für Alttestamentliche Exegese neben den Vorlesungen an der Theologischen Fakultät, Sektion Tarnów, auch Vorlesungen und Übungen an anderen Instituten durch. Vorlesungen zur Einführung und zur Exegese einzelner Bücher des Alten Testaments finden in Tochów statt - für die Schwestern und die Klostergemeinschaft. Die wissenschaftlichen Forschungsarbeiten konzentrieren sich auf die Vorbereitung von Vorträgen über Themen aus dem Alten Testament.

Inähnlicher Weise arbeiten die Mitarbeiter des Lehrstuhls für Neutestamentliche Exegese an Aufgaben aus der Didaktik und an wissenschaftlichen Erhebungen, sowie in Verbindung mit dem „Dzieło Biblijne im. Jana Pawła II” an der Durchdringung der Bibel. Grundsätzlich konzentriert sich der Lehrstuhl auf die Vorbereitung weiterer Vorlesungen und Übungen, wie auch auf zusätzliche Arbeiten mit den Studenten im Rahmen von Bibelbegegnungen, welche die Hörer zu selbstständiger Bibelarbeit befähigen. Im Bereich der Didaktik unterstützen die Mitarbeiter des Lehrstuhls neben den Vorlesungen an der Theologischen Fakultät, Sektion Tarnów, ein Priesterseminar in der Ukraine und die Weiterbildung der Schwestern in Ordenshäusern (Dębica, Tuchów, Zakliczyn, Tarnów). In Zusammenarbeit mit dem „Dzieło Biblijne im. Jana Pawła II” werden offene Bibelstudien für Laien und Geistliche in Tarnów, Mielec, Dębica und Brzesko angeboten. Diese Veranstaltungen werden zweimal im Monat abgehalten und umfassen Material aus der allgemeinen und ausführlichen Introduktion in das Alte und Neue Testament. Ähnliche Vorträge gibt es für das Bibelstudium im Radio oder im Internet. Darüber hinaus bereitet der Lehrstuhl Symposien vor - das letzte bezog sich auf den Apostel Paulus. Derzeit laufen die Arbeiten an weiteren Ausgaben der Quartalsschrift „Krąg Biblijny” (Bibelkreis) des „Dzieło Biblijne im. Jana Pawła II".

Das Hauptinteresse der Mitarbeiter am Lehrstuhl für Patrologie und Kirchengeschichte liegt auf dem umfassenden Verstehen der Kirchengeschichte, unter besonderer Berücksichtigung der Anfangszeit des Christentums. Im Rahmen der Erforschung des Urchristentums ist eine genaue Spezifikation kleinerer Untersuchungsbereiche notwendig, da die Patrologie ihrer Bedeutung nach alles umfasst, was mit dem Christentum und mit dem Kontext in Verbindung steht, in dem es entstanden ist. Dagegen nimmt die Kirchengeschichte den gesamten Zeitraum 
von den Anfängen des Christentums bis in die heutige Zeit in den Blick. Da sich ein einzelner Lehrstuhl kaum auf intensive Art und Weise mit der gesamten Patrologie und Kirchengeschichte befassen kann, werden in Abhängigkeit der Möglichkeiten und des Bedarfs Prioritäten gesetzt. Den Schwerpunkt der Forschungsarbeiten bilden Patrologie und Kirchengeschichte des Frühchristentums. Diese Priorität ergibt sich aus der Bedeutung der patristischen Theologie und der Bedeutung der damals errichteten Institutionen. Beachtenswert ist auch, dass sich die Mitarbeiter des Lehrstuhls in dieser Materie spezialisieren. Aktuelle Arbeiten befassen sich mit dem breiten Spektrum der wichtigen Fragen zur frühchristlichen Initiation (Doktrin, Praxis Institutionen, etc.). Dieser Thematik widmen sich ebenso die Seminare, aus denen sich die Magisterarbeiten entwickelt haben. Ein weiterer bedeutender Forschungsschwerpunkt der letzten Jahre war die neuere Geschichte, insbesondere die Zeit der kommunistischen Diktatur. Der Lehrstuhl untersucht dazu Schicksale der Diözese in der Phase des Kommunismus. Diese Untersuchungen sind sowohl im Hinblick auf die Dokumentation als auch die Didaktik von großer Bedeutung. Dieser für die Geschichte der Diözese Tarnów so wichtige Zeitraum, dessen Auswirkungen in vielen Fällen noch heute spürbar sind, soll besonders den Seelsorgern bekannt sein, die ihre Arbeit gegenwärtig aufnehmen. Sie müssen mit dem Wissen über die gesamte Diözese und einzelne Pfarreien vertraut sein. Daher bilden Fragen aus diesem Fachgebiet insbesondere in den wissenschaftlichen Seminaren das Hauptobjekt der Beschäftigung.

Am Lehrstuhl für Fundamentaltheologie und vergleichende Religionswissenschaft (Ökumenismus) suchen die Mitarbeiter nach einer eigenen Sprache, mit der die grundlegenden christlichen Glaubenswahrheiten besser weiter gegeben werden können. Hierbei handelt es sich um eine besonders dringliche Aufgabe, wenn es darum geht, komplizierte Themen „menschlich” zu erläutern, wie etwa Kataklysmen in der Natur oder Unglücksfälle, in denen einzelne Menschen oder sogar eine ganze Personengruppe umkommen. Daher befasst sich das Projekt damit, die theologische Begründung in dem Bereich der kirchlichen Lehre zu vertiefen, der die geheimnisvollen Wege der Göttlichen Vorsehung betrifft. Ausgehend von der gläubigen Erkenntnis, dass Gott will, der Mensch möge die offenbarte Wahrheit kennen lernen und annehmen, bemüht sich die Fundamentaltheologie darum, die nötigen Anstrengungen zu unternehmen, um die Wahrheit über Gottes Vorsehung im Licht der Göttlichen Liebe darzustellen.

Die akademischen Mitarbeiter am Lehrstuhl für Dogmatik untersuchen die breit gefassten Grundlagen der kirchlichen Lehre, die sich im Bekenntnis des Glaubens sowie in dessen Raum und Bedeutung im Leben der Gläubigen ausdrückt. Dabei gilt das Interesse der Lehrstuhlmitarbeiter besonders den Verbindungen der Doktrin mit 
dem kulturellen Leben, das sowohl unter historischem Aspekt als auch im Kontext der aktuellen Herausforderungen und Bedürfnisse betrachtet wird, die im kirchlichen Leben zu notieren sind. Auf diese Art und Weise können die grundlegenden und in der Tradition fest verwurzelten Fragestellungen mit den neu auftretenden Bedürfnissen verknüpft werden. Diese Problematik steht in unmittelbarer Verbindung mit den didaktischen Aufgaben, welche die Mitarbeiter des Lehrstuhls erfüllen. Es ist bereits gelungen, viele der durchgeführten Untersuchungen in vorgelegten Publikationen zugänglich zu machen, womit die Bedeutung der Forschungsarbeiten bestätigt und auf die Zielrichtung verwiesen wird, die in weiteren Arbeiten aufgegriffen werden kann. All dies belegt, dass diejenigen Forschungen immer wichtiger werden, die sich mit den Verbindungen zwischen dem christlichen Glauben und der Kultur befassen. Die Arbeiten basieren auf einem allgemeinen Forschungsprogramm, das sich über einige Jahre hinweg erstreckt.

Am Lehrstuhl für Moraltheologie und Geistlichkeit greifen die Mitarbeiter im Rahmen ihrer wissenschaftlichen Untersuchungen die aposteriorischen Probleme auf, die sich angesichts der Erwartungen des Menschen ergeben, der heute in sich ständig verändernden Bedingungen leben muss. In einer Situation, in der für das Wohl des Individuums und der Gesellschaft häufig frappierende Handlungsvorschläge und -muster vorgegeben werden, akzentuieren derartige Untersuchungen die unveränderlichen Grundsätze, die aus dem natürlichen Recht, dem Dekalog, den Evangelien und der Soziallehre der Kirche hervorgehen. Gegenwärtig konzentrieren sich die Forschungen der Mitarbeiter auf die Identität von Ehe und Familie in der heutigen Zeit sowie auf die Entwicklung ihrer Geistlichkeit. Diese Aufgabe ist gerade jetzt von außerordentlicher Bedeutung, wenn man die vielfältigen Attacken auf die Familie betrachtet, die insbesondere aus den modernen Kommunikationsmedien kommen.

Die Mitarbeiter des Lehrstuhls für Religionspädagogik und Katechese befassen sich mit Problemen, die mit dem Prozess einer integralen Erziehung des Menschen unter besonderer Berücksichtigung seiner religiösen Erziehung in Verbindung stehen. Die gesellschaftlichen, politischen, kulturellen, ökonomischen und religiösen Herausforderungen veranlassen alle an Erziehung und Bildung beteiligten Institutionen und Milieus dazu, sowohl nach neuen Modellen als auch nach innovativen Lösungen in diesem Bereich zu suchen. Unter Berufung auf die gegenwärtige Lehre der Kirche, insbesondere auf die Lehre Papst Johannes Pauls II., auf moderne Katechesemodelle und auf aktuelle empirisch ermittelte Daten aus der Soziologie und der Psychologie führen die Mitarbeiter des Lehrstuhls Forschungen durch, um nach neuen Modellen für die christliche Erziehung in Familie, Schule und Pfarrgemeinde zu suchen, die den Bildungsprozess einer 
persönlichen und religiösen Reife des Menschen begünstigen. An diesem Lehrstuhl werden bereits seit einigen Jahren in der Zusammenarbeit mit anderen Hochschulen in Polen und ganz Europa Forschungsarbeiten aufgenommen, deren Ziel nicht nur in der Diagnose der Situation besteht sondern ebenso in der Ausarbeitung neuer Modelle für die Katechese von Kindern im Vorschulalter und im Schulalter, daneben auch neuer Modelle für die Familienkatechese, für die Zusammenarbeit aller Erziehungs- und Bildungsumgebungen sowie für die Pädagogik. Weil die Diözese Tarnów über reichhaltige Erfahrungen auf diesem Gebiet verfügt (z.B. die Katechese für die Vorschule, die Schulkatechese und die Katechese zur Ehevorbereitung), bereitet der Lehrstuhl auf der Basis der in den empirischen Untersuchungen erhobenen Daten und der von polnischen Katecheten festgelegten theoretischen Grundlagen pädagogische Lösungen, die dem modernen Kontext und den Rahmenbedingungen der heutigen Zeit adäquat sind. Die Ergebnisse der bisherigen Forschungen finden sich in Lehr- und Schulbüchern sowie in monografischen Arbeiten (im Team) wieder. Die im Rahmen der Statuten dotierten Finanzen ermöglichen eine intensive Forschungsarbeit in diesem Bereich. In der Zusammenarbeit mit deutschen, flämischen, slowakischen, slowenischen, portugiesischen, tschechischen, englischen, litauischen, ungarischen, bulgarischen und irischen Hochschulen nehmen die Mitarbeiter des Lehrstuhls Forschungsprojekte auf, die sich mit der religiösen Erziehung in der Familie in ausgewählten Ländern Europas befassen und insbesondere den interreligiösen Aspekt berücksichtigen. Diese Forschungsarbeiten des Lehrstuhls tragen auch dazu bei, die Innovationen und die Konkurrenz in der Volkswirtschaft zu erhöhen, da sie Fragen zur Qualitätsverbesserung von religiöser Erziehung und Katechese erörtern, was insgesamt die Entwicklungsmöglichkeiten des menschlichen und gesellschaftlichen Kapitals im Land beeinflusst.

Der Forschungsschwerpunkt am Lehrstuhl für Kanonisches Recht lag in den letzten Jahren auf Problemstellungen im kirchlichen Verfahrensrecht (Kodex des Kanonischen Rechts, siebtes Buch, aus dem Jahr 1983) sowie im Kanonischen Eherecht. Dabei handelt es sich einerseits um rein theoretische Fragen (sie gehen in die Themenstellung der Vorlesungen ein), andrerseits sind sie auch praktischer Art (vor allem auf dem Gebiet der Seelsorge - da Ehevorbereitungskurse, wie kanonische Eheprozesse ebenso eine seelsorgerische Dimension aufweisen). Der Erfolg der Arbeit am Lehrstuhl lässt sich an vier landesweite Symposien in den Bereichen Eherecht und kirchliches Verfahrensrecht (2004, 2005, 2007 und 2009) erkennen, die vor allem für die Mitarbeiter der kirchlichen Gerichtsbarkeit in Polen organisiert wurden. Dabei waren insbesondere das zweite und das dritte Symposium der Verfahrensinstruktionen des Apostolischen Stuhls „Dignitas connubii” 
gewidmet. Für die nähere Zukunft wurden weitere Forschungen geplant, die sich vor allem auf die Analyse der Urteile des apostolischen Gerichts der Römischen Rota (Rota Romana) unter ausgewählten Aspekten des Verfahrensrechts beziehen werden. Am 13. und 14. Juni fand bereits die nächste wissenschaftliche Konferenz in Gródek nad Dunajcem statt, das fünfte landesweite Gerichtsforum: „Wady oraz zalety kanonicznego procesu o stwierdzenie nieważności małżeństwa" (Mängel und Vorzüge im Kanonischen Prozess der Ehenichtigkeitsverfahren). Einen anderen Untersuchungsbereich des Lehrstuhls für Kanonisches Recht bildet darüber hinaus die kontinuierliche Beschäftigung mit Fragen rund um die Problematik der Familie und die Rechtsposition von Menschen, die in Beziehungen außerhalb des Ehesakraments zusammenleben. Außerdem werden Probleme untersucht, die mit dem Bekenntnisrecht zusammenhängen, z.B. das karitative Handeln in Übereinstimmung mit dem Kanonischen Recht und dem Bekenntnisrecht.

Das Wirken der Mitarbeiter am Lehrstuhl für Sozialwissenschaften, Pastoraltheologie, Liturgie und Homiletik kreist um die Handlungsmethodik, die in der Gesellschaftswissenschaft der Kirche allgemein aufgenommen und anerkannt wurde, also um die Triade: sehen, bewerten, handeln. Deshalb konzentrieren sich die wissenschaftlichen Forschungen auf die Beschreibung und die Diagnose der Veränderungen innerhalb der Gesellschaft. Da keine Veränderung als einzig als Fortentwicklung zu charakterisieren ist, müssen die derzeit stattfindenden Umbrüche kritisch diagnostiziert werden. Die Dynamik im gesellschaftlichen Leben erfordert unablässige Reflexion, welche die Reihe moderner Tendenzen mit den unveränderlichen ethisch-sozialen Grundsätzen konfrontiert. Als Effekt dieser Forschungen ergibt sich eine Antwort auf die Frage: Sind die derzeit stattfindenden Veränderungen ein Fortschritt oder ein Regression? Das Reflektieren über die Bewertung der Umbrüche bestimmte die Richtung der Forschungsarbeiten an dem Lehrstuhl. Es besteht Übereinstimmung, dass das wichtigste Kriterium bei allen Veränderungen der Mensch ist. Um dem Kriterium der Würde des Menschen ausreichende Beachtung zu schenken, werden die Untersuchungen dahingehend durchgeführt, dass die gegenwärtigen Umbrüche im Hinblick darauf analysiert werden, wie dem Menschen ein gebührender Platz in dem weitreichenden Prozess erhalten werden kann, in dem sich die gesellschaftlichen, kulturellen, wirtschaftlichen, politischen und viele andere Verhältnisse ständig verändern. Dies bedeutet, dass diejenigen Veränderungen Fortentwicklung bedeuten, welche die Würde des Menschen erhalten, wenn jedoch der Mensch durch die stattfindenden Umbrüche degradiert wird, dann ist von Regression zu sprechen. Diese Forschungsarbeiten umfassen auch die Reflexion über den Menschen im Kontext der Gegenwart. Der Bereich 
dieser Studien wird von den Sozialenzykliken inspiriert, unter denen „Populorum progressio”, „Sollicitudo rei socialis” und „Centesimus annus” anzuführen sind.

Es scheint, das gerade die wissenschaftlichen Forschungen, die von den akademischen Mitarbeitern der Theologischen Fakultät, Sektion Tarnów, der Päpstlichen Universität Johannes Paul II. in Krakau aufgenommen werden, eine kontinuierliche Vertiefung der Göttlichen Offenbarung darstellen, um die Papst Johannes Paul II. in seiner Enzyklika „Fides et Ratio” gebeten hat: „Es drängt mich, diese Enzyklika mit einem letzten Gedanken abzurunden, mit dem ich mich vor allem an die Theologen wende, damit sie den philosophischen Implikationen des Wortes Gottes besondere Aufmerksamkeit schenken und eine Überlegung anstellen, aus der sich die spekulative und praktische Substanz der theologischen Wissenschaft ergibt. Ich möchte ihnen für ihren kirchlichen Dienst danken"2. Die Bischöfe Polens verwiesen in ihrem Hirtenbrief zum Pfingstfest 2005 darauf, wie nötig es ist, das große Erbe zu durchdringen, das der Heilige Vater Johannes Paul II. hinterlassen hat: „Für dieses große Gut müssen wir Gott von Herzen danken und darum bitten, dass Er uns hilft, es zu bewahren und zu entwickeln. Am heutigen Tag, an dem - nach fast 27 Jahren segensreicher Dauer - das Pontifikat des großen Papstes endet, müssen wir dem gewaltigen Erbe von Herzen Achtung schenken, das er uns hinterlassen hat. Wie viele und wie bedeutende Wahrheiten hat er uns in seinen Enzykliken, Adhortationen, Apostolischen Briefen, Homilien und Katechesen weiter gegeben! Wie viele lebenswichtige Ermutigungen, Aufforderungen und Ermahnungen hat er an uns Polen während seiner achtfachen Pilgerreisen in sein Vaterland gerichtet! Nicht immer haben wir sorgfältig genug auf ihn gehört, häufig haben wir seine Anwesenheit mehr erlebt als seine Worte, weil wir Gedanken und Herzen nicht an die Tiefe seiner evangelischen Verkündigung geheftet haben. Jetzt ist die Zeit dafür gekommen, im Geist des Glaubens die Überlegung aufzunehmen, deren Frucht nicht nur mehr Kenntnis über seine Lehre sein wird sondern vor allem ein besseres Leben... Wie viele wichtige, kostbare Hinweise - und wie viele Aufgaben liegen vor uns, wenn wir dieses Gnadengeschenk, das für uns der Mensch Johannes Paul II. und seine Lehre waren und bleiben werden, nicht verkommen lassen wollen!”3.

\footnotetext{
${ }^{2}$ Fides et Ratio 105.

${ }^{3}$ Ojciec święty Jan Paweł II - bezcenny i zobowiązujący dar od Boga. List Pasterski biskupów polskich na Uroczystość Zesłania Ducha Świętego 2005 r., „Currenda” (2005) Nr. 2, S. 190-191.
} 